Check for updates

Cite this: RSC Adv., 2017, 7, 28469

\title{
A monolithic air cathode derived from bamboo for microbial fuel cells $\uparrow$
}

\begin{abstract}
Wei Yang, ${ }^{\text {ab }}$ Jun Li, (D)*ab Liang Zhang, (D) ab Xun Zhu ${ }^{\text {ab }}$ and Qiang Liao ${ }^{\text {ab }}$
To simplify the cathode fabrication, we herein report a bamboo charcoal tube-derived (BCT) air cathode for microbial fuel cells (MFCs). This monolithic cathode was prepared by carbonizing a bamboo tube in a $\mathrm{N}_{2}$ atmosphere at $900{ }^{\circ} \mathrm{C}$. The scanning electron microscope indicates that the porous structure of the $\mathrm{BCT}$ cathode can provide possible channels for oxygen supply and proton transfer, and therefore can offer the formation of the triple phase interfaces required for oxygen reduction reaction (ORR) in the bulk of $B C T$. It is also shown that compared to the cathode with bamboo charcoal powder (BC) and Pt/C, the BCT cathode exhibited the lowest oxygen mass transfer coefficient of $4.5 \times 10^{-5} \mathrm{~cm} \mathrm{~s}^{-1}$. Additionally, the rotating ring-disk electrode tests demonstrate that the BCT cathode exhibited a considerable activity toward ORR. Consequently, the MFC with BCT delivered a comparable maximum power density $(40.4 \pm$ $\left.1.5 \mathrm{~W} \mathrm{~m}^{-3}\right)$ to that with $\mathrm{Pt} / \mathrm{C}\left(37.7 \pm 2.5 \mathrm{~W} \mathrm{~m}^{-3}\right)$ and the highest coulombic efficiency of $55.87 \pm 1.0 \%$ as compared with the MFCs with BC $(29.9 \pm 0.4 \%)$ and $\mathrm{Pt} / \mathrm{C}(17.2 \pm 0.4 \%)$. These results indicate that BCT could serve as a low cost, simple fabrication and high-performance alternative cathode for MFCs.
\end{abstract}

Received 24th April 2017

Accepted 18th May 2017

DOI: $10.1039 / c 7 r a 04571 a$

rsc.li/rsc-advances

ORR in MFCs. For example, Liu et al. reported a maximum

\section{Introduction}

In the areas of wastewater treatment and electricity generation, microbial fuel cells (MFCs) are a "green" bio-electrochemical technology that can simultaneously degrade organic matter by the metabolic of bacteria and generate bioelectricity. ${ }^{\mathbf{1 - 4}}$ MFCs with air-cathode have the greatest potential for practical utilization due to their simple design and direct use of oxygen in the air as electron acceptor. As one of the most important factors influencing MFC performance, air-cathode has recently attracted increasing attention. ${ }^{5}$

The performance of air cathodes is limited due to the high over-potential and sluggish oxygen reduction reaction (ORR) under neutral $\mathrm{pH}$ conditions. ${ }^{6}$ The traditional platinum catalyst is commonly used as the ORR catalyst because of its high electrocatalytic activity. ${ }^{7}$ However, the high price hinders feasibility in practical utilization. State-of-the-art studies have demonstrated that the carbonaceous catalysts derived from biomass, e.g., cellulose phosphate, natural leaves, and blood protein, were promising alternatives to Pt due to their low cost, high specific surface area and abundance. ${ }^{8-10}$ Several successful applications of these ORR catalysts have been previously demonstrated their considerable electrocatalytic activity toward

${ }^{a}$ Key Laboratory of Low-grade Energy Utilization Technologies and Systems, Chongqing University, Chongqing 400030, China. E-mail: lijun@cqu.edu.cn; Fax: +86-23-65102474; Tel: +86-23-6510-2474

${ }^{b}$ Institute of Engineering Thermophysics, Chongqing University, Chongqing 400030, China

$\dagger$ Electronic supplementary information (ESI) available. See DOI: 10.1039/c7ra04571a power density of $2293 \pm 50 \mathrm{~mW} \mathrm{~m}^{-2}$ with a catalyst derived from cellulose-derived N- and P-doped carbon. ${ }^{11}$ Similarly, Zhou et al. synthesized a carbonaceous catalyst by pyrolyzing natural spider silk as a precursor, and they obtained a high power density of $1800 \pm 82 \mathrm{~mW} \mathrm{~m}^{-2} \cdot{ }^{12}$ These catalysts have a comparable or even higher catalytic performance compared to that of $\mathrm{Pt}$ in neutral $\mathrm{pH}$ due to the presence of the high amount of $\mathrm{N}$ and/or $\mathrm{P}$ containing surface groups and their tolerance to the poisoning species in MFC relevant conditions. ${ }^{\mathbf{1 3 , 1 4}}$

Alternatively, conventional air cathodes for MFCs consist of several functional layers, and they are usually fabricated by a complex powder-based route. Typically, the mixture of ORR catalysts and binders (e.g., Nafion and/or PTFE) was applied on one side of a supporting electrode and served as the catalyst layer (CL). The carbon black and polytetrafluoroethylene (PTFE) suspension was then coated on the other side of the supporting electrode as the gas diffusion layer (GDL). For example, Cheng et al. prepared the air cathode by brushing the Pt catalyst on the water-facing side of the carbon cloth and the mixture of carbon black and $30 \mathrm{wt} \%$ PTFE solution onto the air-facing side of the carbon cloth, achieving a maximum power density of $766 \mathrm{~mW}$ $\mathrm{m}^{-2} \cdot{ }^{15}$ Zhang et al. developed a method by pressing the mixture of carbonaceous catalysts and PTFE on one side of a metal mesh, a MFC with the prepared cathode had a maximum power density of $1220 \mathrm{~mW} \mathrm{~m}^{-2}$ compared to $1060 \mathrm{~mW} \mathrm{~m}^{-2}$ of $\mathrm{Pt}$ cathode. ${ }^{16}$ To optimize the triple phase interfaces (TPIs) in CL on water-facing side, Dong et al. proposed the rolling-press method to optimize the cathode structure by $\sim 37 \%$ increase in power density. ${ }^{17} \mathrm{Li}$ et al. further optimized the opening size of 
metal matrix in rolling-pressed activated carbon air cathode to enhance the MFC performance from $1485 \pm 18 \mathrm{~mW} \mathrm{~m}^{-2}$ to 2151 $\pm 109 \mathrm{~mW} \mathrm{~m}^{-2} \cdot{ }^{18}$ From above, it is clear that air cathodes are commonly prepared by brushing and cold/hot pressing methods. However, the brushing method is coarse and laborconsuming, while the pressing method (e.g., hot press) needs complicated procedures (e.g., paste preparation of catalyst and gas diffusion layer, the sequential pressing process). Recently, Chen et al. and Wang et al. prepared a binder-free air cathode using stainless steel mesh as a support, avoiding the use of PTFE in preparation process. ${ }^{19,20}$ However, the complex procedures, such as the preparation of GDL and/or CL separately and their assembly on metal mesh by pressing or rolling press, still hinder its practical utilization.

An air cathode with an easily fabricated design and porous structure, incorporating the merits of low preparation cost for scaling up and high electrocatalytic activity, would make the commercial applications of MFC technology a realistic possibility. Interestingly, bamboo is a functional material composed of hollow vessels surrounded by fibrous sclerenchyma cells. ${ }^{21,22}$ After carbonization, vascular bundles and parenchyma cells in bamboo stems could evolve into pores. These pores can provide possible channels for oxygen supply and proton transfer, and therefore can offer possible formation of TPIs for ORR. In our previous study, it was demonstrated that bamboo charcoal (BC) powder prepared by carbonizing bamboo stems in a nitrogen atmosphere had a high electrocatalytic activity and poison tolerance towards ORR in MFC relevant conditions. ${ }^{\mathbf{1 4}}$ The bamboo charcoal tube (BCT), derived from the same precursor as bamboo charcoal powder, should also have the similar ORR activity and can be directly used for catalyzing ORR without the involvement of the PTFE and/or Nafion binder. Meanwhile, the inherent porous and tubular structure in BCT can serve as oxygen or proton transfer channels, and the monolithic structure support, respectively. In general, an air-cathode is composed of a GDL for oxygen and a CL for proton supply and TPIs, as well as a structure support. Therefore, given the multiple functions of BCT, such as a tubular structure with suitable porosity for proton and oxygen transfer and high catalytic activity for ORR, the BCT has the potential as the architecture prototype of an air cathode, which allowed an easier fabrication process for the monolithic cathode.

In this paper, a carbonized bamboo tube was used as the air cathode of an MFC. The textural properties of the BCT cathode were investigated by scanning electron microscopy (SEM). The oxygen permeability of the BCT cathode was studied by comparing the oxygen mass transfer coefficient $\left(K_{\mathrm{O}_{2}}\right)$ with the cathodes made by the conventional powder-based method using BC powder and the commercial $\mathrm{Pt} / \mathrm{C}$ as the catalysts. The ORR catalytic activity of BCT was also confirmed using a rotating ring-disk electrode (RRDE) and linear sweep voltammetry (LSV) in the growth medium discharged from an operating MFC. Based on the number of electrons transferred, the reaction pathway towards ORR of the catalyst was discussed. Additionally, the performance of the cathode was evaluated to confirm the feasibility of its application in MFC technologies.

\section{Methods and materials}

\subsection{Preparation of the air cathodes}

The bamboo charcoal tube (BCT) electrode is directly derived from a bamboo tube (Phyllostachys pubescens Mazel). In detail, bamboo tubes were collected from the south of China and were washed with deionized water and dried at $80{ }^{\circ} \mathrm{C}$ for 3 days. The dried bamboo tubes were used as the precursors for BCTs, which were directly carbonized in a tube furnace (JQF1100-30, China) at $900{ }^{\circ} \mathrm{C}$ at a $\mathrm{N}_{2}$ flow rate of $100 \mathrm{~cm}^{3} \mathrm{~min}^{-1}$, followed by heat treating at $350{ }^{\circ} \mathrm{C}$ for $2 \mathrm{~h}$ under an air atmosphere to increase the porosity. The schematic of the preparation process and the microstructure of the carbonized BCTs are shown in Fig. 1a. Then, the prepared BCT was polished with sandpaper to form a shape with a thickness of $3.0 \pm 0.2 \mathrm{~mm}$, an external diameter of $40 \pm 1 \mathrm{~mm}$ and a height of $50 \pm 1 \mathrm{~mm}$. To remove the possible metal impurities inherently contained in BCT, the shaped sample was soaked in $1 \mathrm{M} \mathrm{HCl}$ solution overnight and was washed with deionized water, and then dried at $80^{\circ} \mathrm{C}$. The monolithic BCT cathode was prepared by brushing PTFE solution (60 wt\%, Hesen, Shanghai, China) on the external surface of BCT to serve as the water-proof layer. After drying at room temperature, it was heat-treated at $340{ }^{\circ} \mathrm{C}$ for $30 \mathrm{~min}$. To compare the performance of an MFC equipped with the BCT cathode, two single-chamber tubular MFCs using perforated polyvinyl chloride tube supported cathode were fabricated with Pt/C (20 wt\%, Alfa Aesar Chemical Co., LTD, China) and the BC powder catalysts, respectively (similar to the fabrication as previous described) (Fig. S1 $\dagger$ ). ${ }^{23}$ The BC powder catalyst was prepared by grounding the $\mathrm{HCl}$-washed BCT sample and sieving the obtained sample with a 2000 mesh sieve. The catalyst loading of the MFCs was $0.5 \mathrm{mg} \mathrm{cm} \mathrm{cm}^{-2}$ for Pt and $80 \mathrm{mg} \mathrm{cm}$ (the same catalyst loading as BCT) for BC, respectively.

\subsection{Physical and chemical analyses}

The X-ray photoelectron spectroscopy (XPS) spectra were acquired using a Thermo Fisher ESCALAB 250Xi spectrometer with a $150 \mathrm{~W}$ monochromatic Al K source. To provide chemical environmental information, a narrow scan was performed for certain elemental core levels with a pass energy of $30 \mathrm{eV}$ to obtain high-resolution spectra. The obtained data were

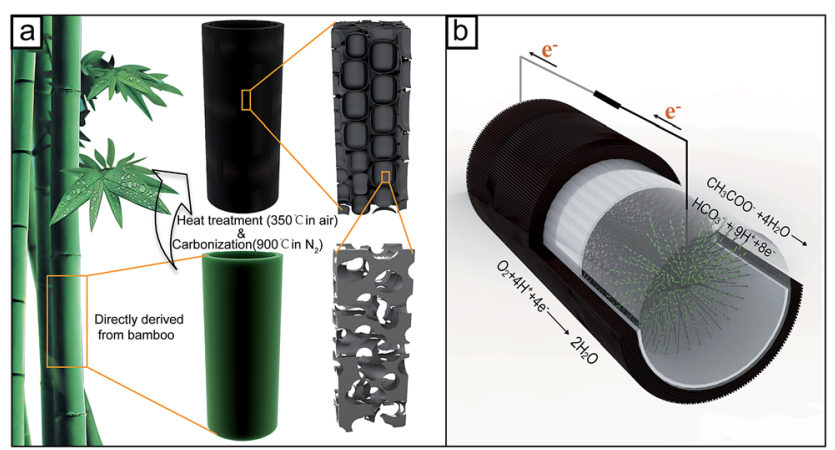

Fig. 1 Schematic for the preparation process of the BCT cathode and the microstructure formed (a), and an MFC with the BCT cathode (b). 
analyzed using the Avantage program provided by Thermo Fisher Scientific Corporation.

The microstructure of the BCT cathode, including crosssectional and longitudinal section, was obtained using a JEOL JSM-7800F SEM. The air/electrolyte/catalyst TPIs of the BCT cathode were evaluated by elemental mapping of fluorine and palladium collected by a scanning transmission electron microscopy (STEM) detector and an Oxford energy dispersive spectrometer (EDS). Fluorine originated from PTFE was used as the indicator for the gas phase in the BCT cathode not only because the out layer of BCT made by PTFE is highly hydrophobic, but also PTFE contains high content of fluorine. To obtain the distribution of the liquid phase inside the BCT cathode, the interior of the sample was first filled by $1 \mathrm{wt} \%$ $\mathrm{PdCl}_{2}$ solution for $8 \mathrm{~h}$ at room temperature to allow the sufficient absorption of $\mathrm{Pd}^{2+}$ into the BCT wall, and then it was washed with $1 \mathrm{M} \mathrm{HCl}$ and distilled water three times and dried in an oven before testing. Due to the absence of inherent palladium in BCT, the exogenous palladium can be used to give the location of liquid phase.

The oxygen permeability of the cathodes was evaluated by comparing the oxygen mass transfer coefficient $\left(K_{\mathrm{O}_{2}}\right)$. The $K_{\mathrm{O}_{2}}$ was calculated from a mass balance on the dissolved oxygen concentration $\left(D_{\mathrm{O}_{2}}\right)$ in the anode chamber over time. The change in $D_{\mathrm{O}_{2}}$ value over time was tracked using dissolved oxygen meters (SG9, Mettler Toledo Instruments Co., Ltd., Shanghai, China), in which the probe was vertically fixed in the center of the anode chamber. Before measurement, the deionized water used for $K_{\mathrm{O}_{2}}$ evaluation was sparged with high purity $\mathrm{N}_{2}(99.99 \%)$ for at least $40 \mathrm{~min}$ to remove the dissolved oxygen. The deaerated water was then injected into the anode chamber, and the $D_{\mathrm{O}_{2}}$ value was recorded continuously for $10 \mathrm{~h}$ after exposure to air, $K_{\mathrm{O}_{2}}$ is calculated according to the method described by Zhang et al. ${ }^{24}$

To evaluate the mechanical strength of the BCT cathode, a fully assembled MFC was connected to a rubber tube. The height of water in tube was gradually increased until the BCT cathode was broken or the water leakage were observed on the outer side of the cathode. The static pressure head $(\mathrm{cm})$ was calculated as the difference in height between the cathode and the top of the water in the tube. ${ }^{5}$

\subsection{Electrochemical analyses}

The electrochemical measurements were performed using an electrochemical workstation (ParSTAT MC, Princeton, USA). A Pt foil was served as the counter electrode and a ring-disk electrode was the working electrode. The reference electrode was an $\mathrm{Ag} / \mathrm{AgCl}$ (saturated with $\mathrm{KCl}$ ) electrode. All potentials were quoted to the $\mathrm{Ag} / \mathrm{AgCl}$ reference electrode. To compare the ORR activity, the HCl-washed BCT was firstly ground and was sieved with a 2000 mesh sieve. Then, $32 \mathrm{mg}$ of the obtained sample and the commercial $\mathrm{Pt} / \mathrm{C}$ was dispersed in a mixture containing $1.9 \mathrm{~mL}$ isopropyl alcohol and $0.1 \mathrm{~mL}$ Nafion (5 wt\% solution, Hesen, Shanghai, China), followed by mixing and ultrasonication for $15 \mathrm{~min}$. The prepared ink $(10 \mu \mathrm{L})$ was dropped onto a $4 \mathrm{~mm}$-diameter glassy carbon (GC) electrode
(BAS. inc., Japan) disk and dried at room temperature. Before coating, the disk was prepared by polishing with $50 \mathrm{~nm}$ alumina paste and cleaned in an ultrasonic bath for $30 \mathrm{~min}$. To simulate the real MFC conditions, the RRDE tests (RRDE-3A, BAS. inc., Japan) were conducted first in nitrogen-sparged growth medium to obtain the baseline current, and then the tests were completed in an air-sparged solution. The growth medium $(\mathrm{pH}$ $=7$ ) discharged from an operating MFC was air-sparged for at least 40 min before RRDE tests, and the gases were continuously injected into the headspace of the electrochemical cell during the experiment. Polarization from -0.6 to $0.3 \mathrm{~V} v s$. $\mathrm{Ag} / \mathrm{AgCl}$ at $100 \mathrm{mV} \mathrm{s}^{-1}$ was conducted to remove possible contaminants or excess oxygen trapped in the pores of the catalyst until the current response of each cycle was the same. For the LSV tests, the potential of the working electrode was scanned from -0.6 to $0.3 \mathrm{~V} v s . \mathrm{Ag} / \mathrm{AgCl}$ at $10 \mathrm{mV} \mathrm{s}^{-1}$ at a rotation rate of $2500 \mathrm{rpm}$, while the potential of the platinum ring was held constant at $0.62 \mathrm{~V} v s$. $\mathrm{Ag} / \mathrm{AgCl}$ for $\mathrm{H}_{2} \mathrm{O}_{2}$ oxidation, as previous described. ${ }^{25}$ To ensure that the current in the current-potential plot only contain faradic currents related to ORR, the current obtained under nitrogen sparging was subtracted from that obtained under oxygen sparging. The average number of electrons transferred $(n)$ in the ORR at the disk electrode was calculated based on the amount of $\mathrm{H}_{2} \mathrm{O}_{2}$ detected using

$$
n=\frac{4 i_{\text {disk }}}{i_{\text {disk }}+i_{\text {ring }} / N_{\mathrm{r}}}
$$

where $i_{\text {disk }}$ is the reduction current at the disk, $i_{\text {ring }}$ is the oxidation current at the ring, and $N_{\mathrm{r}}(0.37)$ is the collection efficiency of Pt ring.

To evaluate the ORR activities of the prepared air-cathodes, LSV tests were conducted in MFC reactors, and the potential of the working electrode was scanned from -0.4 to $0.3 \mathrm{~V} v s$. Ag/ $\mathrm{AgCl}$ at a scan rate of $1 \mathrm{mV} \mathrm{s}^{-1}$. Electrochemical impedance spectroscopy (EIS) of the air cathode was recorded over a frequency range of $10 \mathrm{mHz}$ to $100 \mathrm{kHz}$ at the open circuit potential (OCP) with a sinusoidal perturbation signal amplitude of $10 \mathrm{mV}$. The tests were performed in the same reactor using the anode as the counter electrode and an $\mathrm{Ag} / \mathrm{Ag} \mathrm{Cl}$ electrode as the reference electrode; the working electrode was the air cathode. Nyquist plots were used to interpret the spectra. A least-square fitting program (Zview 3.1) was employed to simulate the Nyquist plots according to an equivalent circuit (as shown in Fig. S2†).

\subsection{MFC set-ups}

The single-chamber tubular MFC with the BCT cathode was constructed by directly using the PTFE coated BCT as the cathode (Fig. 1b). A carbon fiber brush $(30 \mathrm{~mm}$ diameter, $40 \mathrm{~mm}$ length) was used as the anode and was placed coaxially in the BCT cathode. The cathodes using the $\mathrm{Pt} / \mathrm{C}$ and the $\mathrm{BC}$ powder catalyst as the ORR catalysts were prepared using the method as described in Section 2.1 and assembled in MFCs using the same way as the BCT. To get a comparable result and to prevent short circuit between the anode and cathode, a polyether sulfone (PES) membrane $(0.8 \mu \mathrm{m}$, Haiyan Xindongfang plasticizing 
technology co., LTD) was wrapped in the inner wall of the MFC with BCT, BC and Pt/C. The MFC was inoculated with the effluent from an acetate-fed MFC with mixed bacteria. The medium was composed of $2.04 \mathrm{~g} \mathrm{~L}^{-1}$ sodium acetate, $11.82 \mathrm{~g}$ $\mathrm{L}^{-1} \mathrm{Na}_{2} \mathrm{HPO}_{4}, 2.32 \mathrm{~g} \mathrm{~L}^{-1} \mathrm{KH}_{2} \mathrm{PO}_{4}, 0.1 \mathrm{~g} \mathrm{~L}^{-1} \mathrm{NH}_{4} \mathrm{Cl}, 0.5 \mathrm{~g} \mathrm{~L}^{-1}$ $\mathrm{NaCl}, 0.1 \mathrm{~g} \mathrm{~L}^{-1} \mathrm{MgSO}_{4} \cdot 7 \mathrm{H}_{2} \mathrm{O}, 15 \mathrm{mg} \mathrm{L}^{-1} \mathrm{CaCl}_{2} \cdot 2 \mathrm{H}_{2} \mathrm{O}$ and 1.0 $\mathrm{mL} \mathrm{L}^{-1}$ trace elements solution. The prepared medium $(\mathrm{pH}=7)$ was sterilized in a high-pressure steam sterilizer (YXQ-LS-75SII, Shanghai Boxun industrial co., LTD, China) and was sparged with high purity $\mathrm{N}_{2}$ for $30 \mathrm{~min}$. All MFCs were operated in fedbatch mode with a $1000 \Omega$ external resistor at $30 \pm 1{ }^{\circ} \mathrm{C}$ in a constant-temperature room. The medium in the MFCs was replaced when the voltage dropped to $<30 \mathrm{mV}$.

The voltages of the MFCs were recorded at $30 \mathrm{~s}$ intervals using a data acquisition system (Agilent 34970A, Agilent Technologies Inc, USA). To obtain polarization and power density curves, the cell voltage was measured by varying the external resistance from $2000 \Omega$ to $10 \Omega$ with a 40 to 60 min time interval to ensure a stable voltage output. The current density and power density were normalized to a projected volume of the MFC (48 $\mathrm{mL}$ ). Chemical oxygen demand (COD) removal efficiency (RE) and coulombic efficiency (CE) of the MFCs was calculated according to the eqn (S1-S3) in ESI. $\dagger$

\section{Results and discussion}

\subsection{Characterization of the BCT cathode}

Generally, bamboo could be viewed as a functional gradient composite material constituted by long and aligned cellulose fibers embedded in a lignin matrix. ${ }^{26}$ From Fig. $2 a$ and b, it is clear that the carbonaceous structure of the BCT cathode mainly consisted of nearly cylindrical pores with a diameter of


Fig. 2 SEM images of BCT (a) cross section and (b) longitudinal section. EDX of element distribution maps of fluorine (c) and palladium (d). Arrow represent the porosity gradient direction, and circles mark the small pores on the wall of the cylindrical pores.
5-15 $\mu \mathrm{m}$ arranged in a honeycomb-like pattern. These cylindrical pores were mainly resulted from the carbonization of the parenchyma tissues in the bamboo stem. There were also many large pores with a diameter of $40-80 \mu \mathrm{m}$ and may originate from the vascular bundles in bamboo stems. The packing density of the pores originated from the vascular bundles became denser and the size decreased from air-facing to water-facing side (as indicated by the white arrow in Fig. 2a), indicating that the gradient porosity structure of the bamboo stem was retained after the carbonization process. ${ }^{27}$ In addition, many small pores (as marked by the red circles in Fig. 2b) with a size of less than 1 $\mu \mathrm{m}$ were also observed on the wall of the cylindrical pores, suggesting that the pores inside the BCT cathode were interlinked.

It is well accepted that abundant TPIs play a crucial role in the cathode performance improvement of the air cathode MFC systems because ORR can only occur at TPIs. Therefore, to evaluate the TPIs inside the BCT cathode, the contact between the electrolyte and air in the porous structure of the BCT cathode was visualized by using fluorine and palladium element as the indicator for liquid and gas phase, respectively. Fig. 2c and d show the typical fluorine and palladium element distribution in a cross section of the BCT cathode, respectively. Fig. 2c reveals that fluorine was largely concentrated on the outer edge of BCT, with some fluorine element detected inside the BCT cathode. This result indicates the penetration of PTFE into the porous structure of the BCT cathode, forming hydrophobic pores for air supply near the outer edge of BCT. Additionally, Fig. 2d demonstrates that palladium concentration in BCT gradually decreased from the water-facing to the air-facing side. Since the cathode marked by palladium (Fig. 2d) represented the wetted area by the electrolyte, the area near the water-facing side (thickness $\sim 800 \mu \mathrm{m}$ ) marked by dense palladium concentration revealed the area fully wetted by the electrolyte. On the other hand, the gradient palladium concentration in the middle of longitudinal section of BCT (thickness $\sim 1500 \mu \mathrm{m}$ ) suggested a gradually decreased wettability of the electrolyte to the BCT cathode due to the presence of PTFE in BCT, indicative of the formation of the triple phase electrode/electrolyte/gas interface. The area near the air-facing side indicated by fluorine was the channels for oxygen supply due to its waterproofed nature. Therefore, the distribution of fluorine and palladium demonstrates the formation of the TPIs in the bulk of the BCT cathode. The extended TPIs within the bulk of the BCT cathode could result in the increased reaction sites toward ORR, and therefore lead to a good cathode performance.

To evaluate the oxygen permeability of different cathodes, the change of $D_{\mathrm{O}_{2}}$ values over time inside the air cathodes was measured and was compared in Fig. 3. It is observed that the $D_{\mathrm{O}_{2}}$ values inside the $\mathrm{Pt} / \mathrm{C}$ and $\mathrm{BC}$ cathode rapidly increased from near $0.3 \mathrm{mg} \mathrm{L}^{-1}$ to a stable level of $\sim 8.0 \mathrm{mg} \mathrm{L}^{-1}$ in $5 \mathrm{~h}$, whereas it required almost $10 \mathrm{~h}$ for $\mathrm{BCT}$ to reach the similar level. Based on the method described by Zhang et al. ${ }^{24}$ the $K_{\mathrm{O}_{2}}$ values of Pt/C, $\mathrm{BC}$ and BCT were calculated as $1.7 \times 10^{-4}, 1.3 \times 10^{-4}$ and 4.5 $\times 10^{-5} \mathrm{~cm} \mathrm{~s}^{-1}$, respectively. The lower $K_{\mathrm{O}_{2}}$ of the BCT cathode reveals a lower oxygen diffusion from the cathode to the anode, 


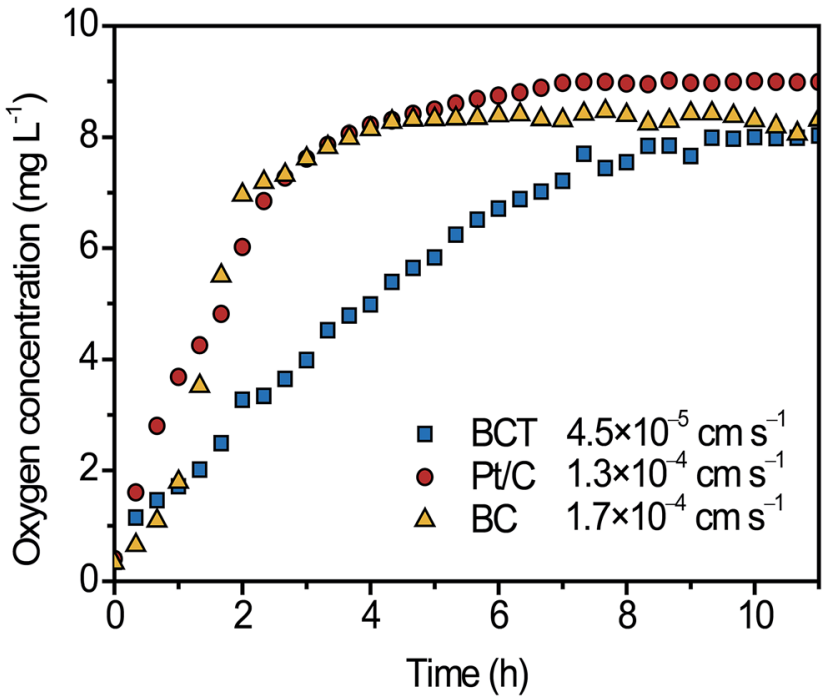

Fig. 3 Change of $D_{\mathrm{O}_{2}}$ value over time for the $\mathrm{BCT}, \mathrm{BC}$ and $\mathrm{Pt} / \mathrm{C}$ cathode.

which would inhibit the aerobic respiration of bacteria to oxide substrates, contributing to the improvement of CE.

The mechanical stability of BCT was verified using the method as previously described. ${ }^{5}$ The BCT cathode showed a good water pressure resistance, tolerating a water pressure head of $\sim 230 \mathrm{~cm}$, which was significantly higher than that of the cathode $(\sim 122 \mathrm{~cm})$ fabricated using PVDF as diffusion layer and a metal mesh as the support. ${ }^{5}$ Results suggested that the BCT cathode had a good mechanical stability in MFC operation.

\subsection{Performance of the MFC with BCT}

To evaluate the power generation of an MFC with the BCT cathode, polarization and power density curves were obtained. As shown in Fig. 4 a, the maximum power density $\left(P_{\max }\right)$ of the MFC with the BCT, $\mathrm{Pt} / \mathrm{C}$ and $\mathrm{BC}$ cathodes was $40.4 \pm 1.5 \mathrm{~W} \mathrm{~m}^{-3}$, $37.7 \pm 2.5 \mathrm{~W} \mathrm{~m}^{-3}$ and $37.1 \pm 2.9 \mathrm{~W} \mathrm{~m}^{-3}$, respectively. The results show that the MFC with the BCT cathode could deliver a comparable $P_{\max }$ to that of Pt/C. Considering the low cost and simple fabrication, the BCT cathode exhibited superiority to Pt/ $\mathrm{C}$, which is usually expensive with a complex fabrication process. In addition, to verify the effect of the anode and cathode performance on the power generation of the MFC, the polarization curves of both electrodes were measured separately. As shown in Fig. 4b, the anode performances were similar, suggesting that the cathode performance of MFC was mainly responsible for enhancing the power output. Additionally, it is clear from Fig. 4b that the BCT demonstrated the highest performance at a high current density higher than 100 $\mathrm{mA} \mathrm{m}{ }^{-3}$. The comparable $P_{\max }$ value and the higher performance of the MFC with BCT at high current densities can be attributed to the higher amount of active sites of BCT resulting from the extended TPIs as compared to that with Pt/C.

$\mathrm{RE}$ and $\mathrm{CE}$ of the MFCs were calculated according to eqn (S1-S3 $\dagger$ ) and are shown in Fig. 4c. The RE of the MFC with BCT was similar to that of the MFCs with $\mathrm{BC}$ and $\mathrm{Pt} / \mathrm{C}$, showing a high (above $80 \%$ ) capacity for wastewater treatment. In contrast to RE, CE varied with the cathodes equipped in the MFCs. As shown in Fig. 4c, the CE was $55.8 \pm 1.0 \%, 29.9 \pm 0.4 \%$ and $17.2 \pm 0.4 \%$ for $\mathrm{BCT}, \mathrm{BC}$ and $\mathrm{Pt} / \mathrm{C}$, respectively. Obviously, the CE of the MFC with BCT was higher than that of the MFC with $\mathrm{BC}$ and $\mathrm{Pt} / \mathrm{C}$. The lower $\mathrm{CE}$ of the MFCs with $\mathrm{BC}$ and $\mathrm{Pt} / \mathrm{C}$ in this study was associated with the higher $K_{\mathrm{O}_{2}}$ value of the BC and $\mathrm{Pt} / \mathrm{C}$ cathodes, which caused more oxygen leakage that allowed the extra substrate consumption by aerobic respiration in the anode. ${ }^{28}$

\subsection{Electrocatalytic properties of the BCT cathode}

As described in Section 2.1 and 2.3, the BC powder catalysts was prepared by grinding and sieving the BCT sample. Therefore, the ORR activity of BC and BCT are assumed to be same in RRDE tests. As a result, we only compare the catalytic activity of $\mathrm{BC}$ and $\mathrm{Pt} / \mathrm{C}$ for ORR. Fig. 5a demonstrated the disk and ring current response of the RRDE measurements at a rotating speed of $2500 \mathrm{rpm}$. Although BC had a more negative onset potential of $-88 \mathrm{mV}$ compared to that of $133 \mathrm{mV}$ for $\mathrm{Pt} / \mathrm{C}$, a comparable limiting current density of $0.98 \mathrm{~mA} \mathrm{~cm} \mathrm{~cm}^{-2}$ was obtained for BC $\left(0.95 \mathrm{~mA} \mathrm{~cm}{ }^{-2}\right.$ for $\left.\mathrm{Pt} / \mathrm{C}\right)$, indicating a similar electrocatalytic activity towards ORR at a potential lower than $-0.3 \mathrm{~V} v \mathrm{vs}$. Ag/ $\mathrm{AgCl}$. The corresponding current responses for the oxidation of hydrogen peroxide ions $\left(\mathrm{HO}^{2-}\right)$ measured by the $\mathrm{Pt}$ ring electrode are shown in Fig. 5a. A slightly higher ring current response for $\mathrm{BC}$ implied that a larger amount of $\mathrm{HO}^{2-}$ was produced on the disk electrode and then reached the ring electrode during the ORR process. Additionally, the average electron transfer numbers $(n)$ of the catalysts at different potentials were calculated based on disk and ring currents from
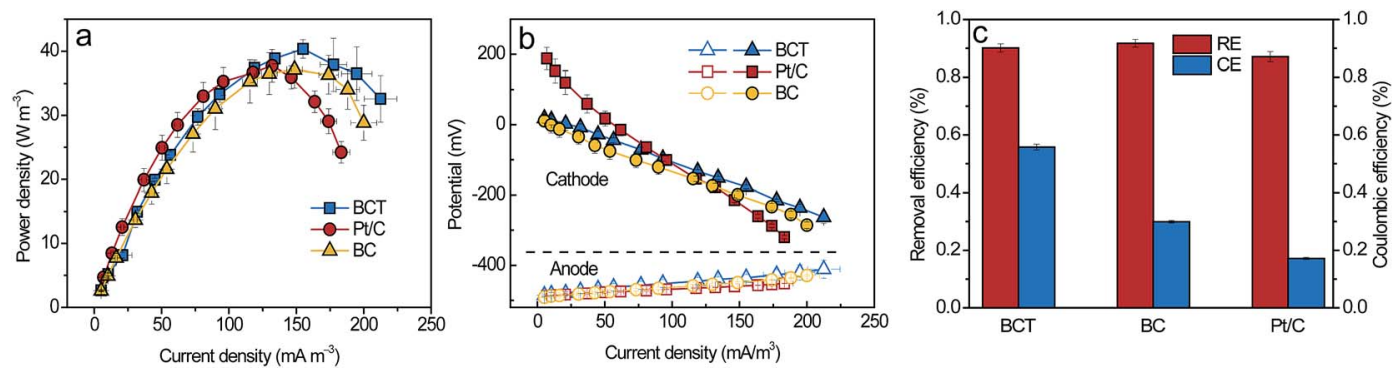

Fig. 4 Power density curves (a), polarization curves (b), RE and CE (at $50 \Omega$ ) (c) of the MFCs with the BCT, BC and Pt/C cathode. 

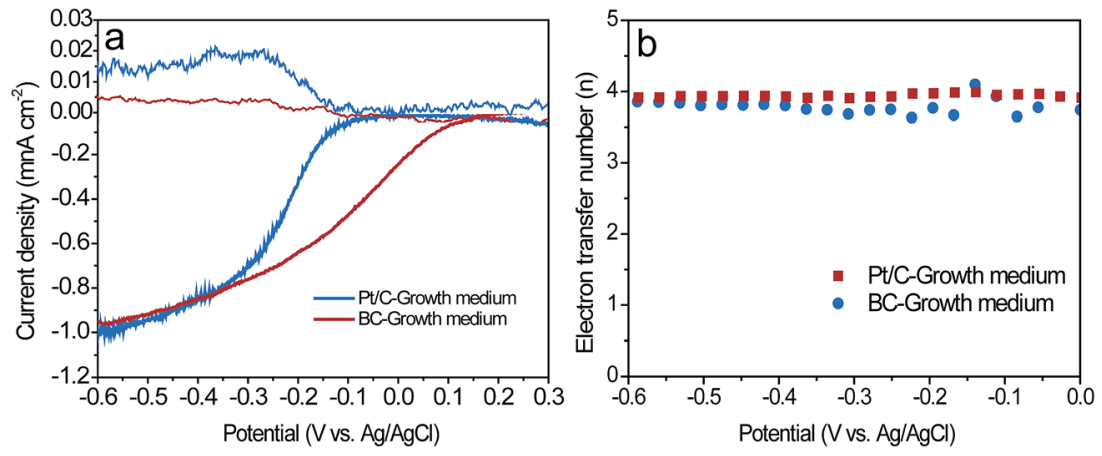

Fig. 5 (a) Disk and ring current response of BC and Pt/C catalysts based on the RRDE measurements in the growth medium at a rotating speed of $2500 \mathrm{rpm}$ and a scan rate of $10 \mathrm{mV} \mathrm{s}^{-1}$. (b) Electron transfer number ( $n$ ) of BC and Pt/C at different potential.


Fig. 6 (a) LSV of the air-cathodes with different catalysts at $1 \mathrm{mV} \mathrm{s}^{-1}$ in an operating air cathode MFC and (b) Nyquist plots of EIS spectra of the air-cathodes (symbols represent experimental data, and lines represent data fitted with the equivalent circuit).

the RRDE measurements (according to eqn (1)). As shown in Fig. 5b, the $n$ values of BC and Pt/C were above 3.6, illustrating a nearly four-electron pathway of ORR. The similar limiting current density and $n$ value indicated that $\mathrm{BC}$ and $\mathrm{Pt} / \mathrm{C}$ had a comparable electrocatalytic activity in the growth medium. Additionally, XPS measurements were used to characterize the chemical composition and the chemical state of heteroatoms in the BC catalyst (Fig. S3, the results were discussed in ESI†). The results indicate that the nitrogen and phosphorus functional groups, such as graphitic-N, pyridinic-N, pyrrolic-N, P-N and C$\mathrm{O}-\mathrm{PO}_{3}$ were formed during the carbonization process. Previous studies reported that graphitic-N and pyridinic- $\mathrm{N}$ can effectively improve the limiting current density and onset potential respectively. ${ }^{29,30}$ Meanwhile, $\mathrm{P}-\mathrm{N}$ and $\mathrm{C}-\mathrm{O}-\mathrm{PO}_{3}$ were also demonstrated as highly reactive and stable active centers for ORR. ${ }^{14,31}$ These functional groups can contribute to the enhancement of ORR catalytic activity.

To evaluate the performance of the cathodes in the MFCs, the LSV of the air cathodes with BCT, BC and Pt/C was recorded at a low scan rate of $1 \mathrm{mV} \mathrm{s}^{-1}$ in the growth medium (Fig. 6a). It is clear that the air cathode based on BCT demonstrated the highest current response in the cathode potential range of -0.6 to $-0.1 \mathrm{~V}$, revealing the superior performance of the BCT cathode. EIS is conducted for the air cathodes in the same electrolyte to determine the resistance of cathode at OCPs. To interpret the EIS results, equivalent circuits were utilized to identify the components of the cathode internal resistances, as previously described (Fig. S2a $\uparrow$ for the $\mathrm{Pt} / \mathrm{C}$ cathode and Fig. S2b $\dagger$ for the BC and BCT cathode). ${ }^{32,33}$ As can be observed in Fig. $6 \mathrm{~b}$ and Table $\mathrm{S} 1, \uparrow$ the ohmic resistance, $R_{\mathrm{o}}$, of the air cathodes was similar for BCT, BC and Pt/C. It is also clear that the charge transfer resistances $\left(R_{\mathrm{ct}}\right)$ of the air cathodes followed the order $\mathrm{BC}>\mathrm{Pt} / \mathrm{C}>\mathrm{BCT}$. The lowest charge transfer resistances $\left(R_{\mathrm{ct}}\right)$ of BCT $(1.74 \Omega)$ can be attributed to the extended TPIs of the cathode, which resulted in the increased reaction sites toward ORR. The higher $R_{\mathrm{ct}}$ of Pt/C, compared to BCT, can be attributed to the deterioration of the catalytic activity of Pt/C toward ORR in the presence of the poisoning species containing in the growth medium. ${ }^{13,14}$

\section{Conclusion}

In this study, a PTFE coated BCT was directly used as the air cathode of an MFC. The BCT cathode obtained by direct carbonization of bamboo tubes in a $\mathrm{N}_{2}$ atmosphere demonstrated a comparable $P_{\max }$ value $\left(40.4 \pm 1.5 \mathrm{~W} \mathrm{~m}^{-3}\right)$ to $\mathrm{Pt} / \mathrm{C}(37.7$ $\pm 2.5 \mathrm{~W} \mathrm{~m}^{-3}$ ) at the same operating conditions. Physical characterization of the BCT cathode indicated that the abundant pores originated from bamboo stems were beneficial for extending the TPIs for ORR, and thus contributed to a higher ORR performance compared to BC. Meanwhile, the lowest $K_{\mathrm{O}_{2}}$ value of the BCT cathode compared with that of $\mathrm{BC}$ and $\mathrm{Pt} / \mathrm{C}$ 
provided a lower oxygen leakage from the cathode to the anode, and therefore led to a comparable power generation with a high $\mathrm{CE}$ of $55.8 \pm 1.0 \%$. These results indicated that BCT could serve as a low cost, simple fabrication and high-performance alternative for the cathode in MFCs.

\section{Acknowledgements}

This work was supported by National Natural Science Funds for Distinguished Young Scholar (No. 51325602), National Natural Science Funds for Outstanding Young Scholar (No. 51622602), the National Science Foundation for Young Scientists of China (No. 51506017) and project supported by the Natural Science Foundation of Chongqing, China (Grant No. cstc2015jcyjA90017).

\section{References}

1 H. X. Chang, Q. Fu, Y. Huang, A. Xia, Q. Liao and X. Zhu, Int. J. Agric. Biol. Eng., 2017, 10, 97-106.

2 J. Li, H. J. Li, J. L. Zheng, L. Zhang, Q. Fu, X. Zhu and Q. Liao, Bioresour. Technol., 2017, 233, 1-6.

3 B. E. Logan and K. Rabaey, Science, 2012, 337, 686-690.

4 S. K. Chaudhuri and D. R. Lovley, Nat. Biotechnol., 2003, 21, 1229-1232.

5 W. Yang, F. Zhang, W. He, J. Liu, M. A. Hickner and B. E. Logan, J. Power Sources, 2014, 269, 379-384.

6 F. Zhao, F. Harnisch, U. Schröder, F. Scholz, P. Bogdanoff and I. Herrmann, Environ. Sci. Technol., 2006, 40(17), 51935199.

7 S. Yang, B. Jia and L. Hong, Bioresour. Technol., 2009, 100, 1197-1202.

8 Q. Liu, S. Chen, Y. Zhou, S. Zheng, H. Hou and F. Zhao, J. Power Sources, 2014, 261, 245-248.

9 S. Y. Gao, X. J. Wei, H. Fan, L. Y. Li, K. R. Geng and J. J. Wang, Nano Energy, 2015, 13, 518-526.

10 C. Z. Guo, C. G. Chen and Z. L. Luo, J. Power Sources, 2014, 245, 841-845.

11 Q. Liu, Y. Zhou, S. L. Chen, Z. J. Wang, H. Q. Hou and F. Zhao, J. Power Sources, 2014, 273, 1189-1193.

12 L. H. Zhou, P. Fu, X. X. Cai, S. G. Zhou and Y. Yuan, Appl. Catal., B, 2016, 188, 31-38.

13 C. Santoro, A. Serov, L. Staryha, M. Kodali, J. Gordon, S. Babanova, O. Bretschger, K. Artyushkova and P. Atanassov, Energy Environ. Sci., 2016, 9, 2346-2353.
14 W. Yang, J. Li, D. Ye, X. Zhu and Q. Liao, Electrochim. Acta, 2017, 224, 585-592.

15 S. Cheng, H. Liu and B. E. Logan, Electrochem. Commun., 2006, 8, 489-494.

16 F. Zhang, S. A. Cheng, D. Pant, G. V. Bogaert and B. E. Logan, Electrochem. Commun., 2009, 11, 2177-2179.

17 H. Dong, H. Yu, X. Wang, Q. Zhou and J. Feng, Water Res., 2012, 46, 5777-5787.

18 X. J. Li, X. Wang, Y. Y. Zhang, N. Ding and Q. X. Zhou, Appl. Energy, 2014, 123, 13-18.

19 S. L. Chen, Y. Chen, G. H. He, S. J. He, U. Schröder and H. Q. Hou, Biosens. Bioelectron., 2012, 34, 282-285.

20 Q. Wang, X. Zhang, R. Lv, X. Chen, B. Xue, P. Liang and X. Huang, J. Mater. Chem. A, 2016, 4, 12387-12391.

21 D. Grosser and W. Liese, Wood Sci. Technol., 1971, 5, 290312.

22 H. A. Khalil, I. U. H. Bhat, M. Jawaid, A. Zaidon, D. Hermawan and Y. S. Hadi, Mater. Des., 2012, 42, 353-368.

23 J. R. Kim, G. C. Premier, F. R. Hawkes, R. M. Dinsdale and A. J. Guwy, J. Power Sources, 2009, 187, 393-399.

24 X. Y. Zhang, S. A. Cheng, X. Wang, X. Huang and B. E. Logan, Environ. Sci. Technol., 2009, 43, 8456-8461.

25 V. J. Watson, C. N. Delgado and B. E. Logan, J. Power Sources, 2013, 242, 756-761.

26 J. Jiang and J. Reinf, Plast. Compos., 2016, 35, 824-833.

27 P. G. Dixon and L. J. Gibson, J. R. Soc., Interface, 2014, 11, 20140321.

28 H. Liu and B. E. Logan, Environ. Sci. Technol., 2004, 38, 40404046.

29 L. Lai, J. R. Potts, D. Zhan, L. Wang, C. K. Poh, C. H. Tang, H. Gong, Z. X. Shen, J. Y. Lin and R. S. Ruoff, Energy Environ. Sci., 2012, 5, 7936-7942.

30 D. Guo, R. Shibuya, C. Akiba, S. Saji, T. Kondo and J. Nakamura, Science, 2016, 351, 361-365.

31 F. Razmjooei, K. P. Singh and J. S. Yu, Catal. Today, 2015, 260, 148-157.

32 C. Portet, G. Yushin and Y. Gogotsi, Carbon, 2007, 45, 25112518.

33 C. Domínguez, F. J. Pérez-Alonso, M. A. Salam, S. A. AlThabaiti, A. Y. Obaid, A. A. Alshehri, J. L. Gómez de la Fuentea, J. L. G. Fierroa and S. Rojas, Appl. Catal., B, 2015, 162, 420-429. 